\title{
INTERVAL EIGENVECTORS OF CIRCULANT MATRICES IN FUZZY ALGEBRA
}

\author{
Helena MYŠKOVÁ \\ Department of Mathematics and Theoretical Informatics, Faculty of Electrical Engineering and Informatics, Technical University of \\ Košice, Němcovej 32, 04200 Košice, Slovak Republic, tel.: +421 55602 2445, e-mail: helena.myskova@ tuke.sk
}

\begin{abstract}
Fuzzy algebra is an algebraic structure in which classical addition and multiplication are replaced by $\oplus$ and $\otimes$, where $a \oplus b=$ $\max \{a, b\}, a \otimes b=\min \{a, b\}$. A vector $x$ is an eigenvector of a matrix $A$ if $A \otimes x=x$.

An interval vector $\mathbf{X}$ and the possible and universal eigenvectors are defined. A necessary and sufficient condition for the possible and universal eigenvectors of a circulant matrix are proved and several examples are given.
\end{abstract}

Keywords: fuzzy algebra, circulant matrix, interval vector, possible eigenvector, universal eigenvector

\section{INTRODUCTION}

Matrices in fuzzy algebra are useful for expressing applications of fuzzy discrete dynamic systems, graph theory, scheduling, medical diagnosis [13], [14] or fuzzy logic programs [7].

Eigenvector of a fuzzy matrix characterize stable states of the corresponding discrete event systems. Investigation of the fuzzy eigenvectors of a given matrix is therefore of great importance. The eigenproblem in fuzzy algebra has been studied by many authors. Interesting results were found in describing the structure of the eigenspace and the algorithms for computing the maximal eigenvector of a given matrix were suggested, see, e.g., [1], [10], [11], [15]. The structure of the eigenspace as a union of intervals of increasing eigenvectors is described in [3].

The eigenproblem of fuzzy matrices and its connection to paths in digraphs were investigated in [1], [6].

The structure of the eigenspace for a special case of socalled circulant matrices is described in [5].

In practice, vector inputs are rather contained in some intervals than exact values. The aim of this paper is to describe the interval eigenvectors of circulant matrices. We define the possible and universal eigenvectors of circulant matrices and give necessary and sufficient conditions for them.

\section{PRELIMINARIES}

The fuzzy algebra $\mathscr{B}$ is the triple $(B, \oplus, \otimes)$, where $(B, \leq)$ is a bounded linearly ordered set with binary operations maximum and minimum, denoted by $\oplus$ and $\otimes$, respectively. The least element in $B$ will be denoted by $O$, the greatest one by $I$.

By $\mathbb{N}$ we denote the set of all natural numbers and by $\mathbb{N}_{0}$ the set $\mathbb{N}_{0}=\mathbb{N} \cup\{0\}$. The greatest common divisor of a set $S \subseteq \mathbb{N}$ is denoted by $\operatorname{gcd} S$. For a given natural number $n \in \mathbb{N}$, we use the notations $N=\{1,2, \ldots, n\}$ and $N_{0}=\{0,1, \ldots, n-1\}$.

For any $n \in \mathbb{N}, B(n, n)$ denotes the set of all square matrices of order $n$ and $B(n)$ the set of all $n$-dimensional column vectors over $B$. The matrix operations over $\mathscr{B}$ are defined formally in the same manner (with respect to $\oplus, \otimes$ ) as matrix operations over any field.

For a given matrix $A \in B(n, n)$, the number $\lambda \in B$ and the $n$-tuple $x \in B(n)$ are the so-called eigenvalue and eigenvector of $A$, respectively, if

$A \otimes x=\lambda \otimes x$.

For $\lambda=I$ equation 11 gets the form

$A \otimes x=x$

The eigenspace $V(A)$ is defined as the set of all eigenvectors of $A$, i.e.,

$$
V(A)=\{x \in B(n) ; A \otimes x=x\} .
$$

\section{EIGENVECTORS OF CIRCULANT MATRICES}

A square matrix is circulant if the input values in every row are the same as the values in the previous row, but they are cyclically shifted by one position to the right. Formally, matrix $A \in B(n, n)$ is circulant if

$$
a_{i j}=a_{k l}
$$

whenever

$$
i-k \equiv j-l \bmod n .
$$

Hence, a circulant matrix $A$ is completely determined by inputs $a_{0}, a_{1}, \ldots, a_{n-1}$ in the first row, i.e.,

$A\left(a_{0}, a_{1}, \ldots, a_{n-1}\right)=\left(\begin{array}{cccccc}a_{0} & a_{1} & a_{2} & \ldots & a_{n-2} & a_{n-1} \\ a_{n-1} & a_{0} & a_{1} & \ldots & a_{n-3} & a_{n-2} \\ \vdots & \vdots & \vdots & & \vdots & \vdots \\ a_{1} & a_{2} & a_{3} & \ldots & a_{n-1} & a_{0}\end{array}\right)$.

For a given circulant matrix $A\left(a_{0}, a_{1}, \ldots, a_{n-1}\right)$ we define a strictly decreasing sequence $M(A)=\left(m_{1}, m_{2}, \ldots m_{s(A)}\right)$ recursively as follows:

$$
m_{r}= \begin{cases}\max \left\{a_{i} ; i \in N_{0}\right\} & \text { for } r=1 \\ \max \left\{a_{i} ; a_{i}<m_{r-1} ; i \in N_{0}\right\} & \text { for } r>1\end{cases}
$$

It is easy to see that $m_{1}>m_{2}>\cdots>m_{s(A)}$ and the length $s(A)$ of the sequence $M(A)$ equals to the number of different values in the first row. We shall use the notation $S(A)=\{1,2, \ldots, s(A)\}$. For each $r \in S(A)$ denote by $P_{r}$ the set of all positions of the value $m_{r}$ in first row, i.e.,

$$
P_{r}=\left\{i \in N_{0} ; a_{i}=m_{r}\right\}
$$


and define the numbers

$d_{r}=\operatorname{gcd}\left(P_{r} \cup\{n\}\right), e_{r}=\operatorname{gcd}\left(d_{1}, d_{2}, \ldots, d_{r}\right)=\operatorname{gcd}\left(e_{r-1}, d_{r}\right) . \quad \max _{k \in N} x_{k} \leq m_{1}$

(3) and

The following two lemmas give necessary conditions for a vector $x$ to be an eigenvector of $A$.

Lemma 3.1. [5] Let circulant matrix $A=$ $A\left(a_{0}, a_{1}, \ldots, a_{n-1}\right)$ be given and $x$ be an eigenvector of $A$. Then $x_{k} \leq m_{1}$ for every $k \in N$.

Lemma 3.2. [5] Let circulant matrix $A=$ $A\left(a_{0}, a_{1}, \ldots, a_{n-1}\right)$ be given and $x$ be an eigenvector of $A$, let $k, l \in N$ and $r \in S(A)$. If $x_{k}<m_{r}$ then the following implications hold true

(i) if $k \equiv l \bmod d_{r}$ then $x_{k}=x_{l}$,

(ii) if $k \equiv l \bmod e_{r}$ then $x_{k}=x_{l}$.

In fact, in the previous lemma the part (ii) implies the part (i). The lemma says that in each eigenvector any entry $x_{k}<m_{r}$ must be repeated after $e_{r}$ positions. The following theorem gives a necessary and sufficient condition for a vector $x$ to be an eigenvector of $A$.

Theorem 3.1. [5] Let $A=A\left(a_{0}, a_{1}, \ldots, a_{n-1}\right)$ be a circulant matrix. A vector $x \in B(n)$ is an eigenvector of $A$ if and only if there is a partition $\mathscr{T}$ on $N$ such that for every class $T \in \mathscr{T}$ there exist $x(T) \in B$ and $r(T) \in S(A)$ satisfying the following conditions

(i) $x_{k}=x(T) \leq m_{1}$ for every $k \in T$,

(ii) $r(T)=\max \left\{r \in S(A) ; x(T)<m_{r}\right\}$,

(iii) $T$ is an equivalence class in $N$ modulo $e_{r(T)}$.

For a given $r \in S(A)$ denote $E^{r}=\left\{1,2, \ldots, e_{r}\right\}$. For each $r \in S(A), i \in E^{r}$ define

$N_{i}^{r}=\left\{k \in N ; k \equiv i \bmod e_{r}\right\}$,

i.e., $N_{i}^{r}$ is the equivalence class modulo $e_{r}$ containing $i \in E^{r}$.

Remark 3.1. Since $e_{r+1} \mid e_{r}$ for each $r \in\{1,2, \ldots, s(A)-1\}$, it follows from the properties of the equivalence relation that for each $i, j \in N$ and $r_{1}, r_{2} \in S(A), r_{1} \leq r_{2}$ either $N_{i}^{r_{1}} \subseteq N_{j}^{r_{2}}$ or $N_{i}^{r_{1}} \cap N_{j}^{r_{2}}=\emptyset$.

The following theorem gives other necessary and sufficient condition for a vector $x$ to be an eigenvector of $A$, which will be used in the next section.

Theorem 3.2. Let $A=A\left(a_{0}, a_{1}, \ldots, a_{n-1}\right)$ be a circulant matrix. A vector $x \in B(n)$ is an eigenvector of $A$ if and only if $\max _{k \in N} x_{k} \leq m_{1}$ and for each $r \in S(A), i \in E^{r}$ such that $\min _{k \in N^{r}} x_{k}<m_{r}$ the equality $x_{t}=x_{s}$ is satisfied for each $t, s \in N_{i}^{r}$.

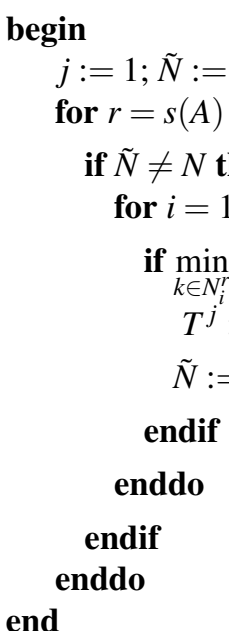
$k \in N_{i}^{j}$ $T^{j} \in \mathscr{T}$

$\max _{k \in N} x_{k}>m_{1}$

or
Proof. Suppose that

We construct a partition $\mathscr{T}$ on $N$ satisfying the conditions from Theorem 3.1 by the following algorithm.

Algorithm $\mathscr{A}$ : Determining a partition $\mathscr{T}$

Input: $A\left(a_{0}, a_{1}, \ldots, a_{n-1}\right)$, vector $x \in B(n)$

Output: the sets $T^{j}$, the elements $x\left(T^{j}\right) \in B$ and the numbers $r\left(T^{j}\right) \in S(A)$

As the output of Algorithm $\mathscr{A}$ we get the sets $T^{1}, T^{2}, \ldots, T^{p}, 1 \leq p \leq n$. We will prove that the sets $T^{1}, T^{2}, \ldots, T^{p}, 1 \leq p \leq n$ create the partition $\mathscr{T}$ on $N$. The inequality $T^{u} \neq T^{v}$ for $u \neq v$ follows from Remark 3.1. Further, for each $l \in N$ there exists $j \in S(A)$ such that $\min _{j} x_{k}<m_{j}$, where $l \in N_{i}^{j}$, i.e., $l \in T^{s}$ for some $s \leq n$, which implies $\underset{k=1, \ldots, p}{\cup} T^{k}=N$.

The validity of the conditions $i$ ) and $i i i)$ of Theorem 3.1 follows from the definitions of $T^{j}$ and $r\left(T^{j}\right)$ for each

The equality $x\left(T^{j}\right)=x_{k}$ for each $k \in T^{j}$ follows from (6) and the inequality in $i$ ) is a consequence of (5). Thus the condition $i$ ) is satisfied for each $T^{j} \in \mathscr{T}$.

By Theorem 3.1 the vector $x$ is an eigenvector of $A$.

For the converse implication suppose that

$(\exists r \in S(A))\left(\exists i \in E^{r}\right)\left[\min _{k \in N_{i}^{r}} x_{k}<m_{r} \wedge\left(\exists t, s \in N_{i}^{r}\right) x_{t} \neq x_{s}\right]$.

If inequality (7) is satisfied then $x$ is not an eigenvector of $A$ by Lemma 3.1

If condition $(8)$ holds true then there exists $r \in S(A), i \in$ $E^{r}$ and $l, m \in N_{i}^{r}$ such that $x_{l}=\min _{k \in N_{i}^{r}} x_{k}<m_{r}$ and $x_{m} \neq x_{l}$. 
Since $l \equiv m \bmod e_{r}$, by Lemma 3.2 i) the vector $x$ is not an

The eigenproblem for circulant matrices in max-plus algebra was studied in [10].

\section{INTERVAL EIGENVECTORS} $\mathbf{X}$.

Similarly to [2], [4], [8], [9] we define an interval vector

Definition 4.1. Let $\underline{x}, \bar{x} \in B(n), \underline{x} \leq \bar{x}$. An interval vector $\mathbf{X}$ with bounds $\underline{x}, \bar{x}$ is defined as follows

$$
\mathbf{X}=[\underline{x}, \bar{x}]=\{x \in B(n) ; \underline{x} \leq x \leq \bar{x}\} .
$$

Definition 4.2. An interval vector $\mathbf{X}$ is

(i) $a$ possible eigenvector of $A$ if there exists $x \in \mathbf{X}$ that is an eigenvector of $A$,

(ii) $a$ universal eigenvector of $A$ if each vector $x \in \mathbf{X}$ is an eigenvector of $A$.

\subsection{Possible eigenvectors}

Theorem 4.1. Let $A=A\left(a_{0}, a_{1}, \ldots, a_{n-1}\right)$ be a circulant matrix. An interval vector $\mathbf{X}$ is a possible eigenvector of $A$ if and only if $\max _{k \in N} \underline{x}_{k} \leq m_{1}$ and for each $r \in S(A), i \in E^{r}$ such that $\min _{k \in N_{i}^{r}} \bar{x}_{k}<m_{r}$, the inequality $\max _{k \in N_{i}^{r}} \underline{x}_{k} \leq \min _{N_{i}^{r}} \bar{x}_{k}$ holds true.

Proof. In formally way, the theorem says that $\mathbf{X}$ is a possible eigenvector of $A$ if and only if

$$
\begin{aligned}
& \max _{k \in N} \underline{x}_{k} \leq m_{1} \\
& \text { and } \\
& (\forall r \in S(A))\left(\forall i \in E^{r}\right)\left[\min _{k \in N_{i}^{r}} \bar{x}_{k}<m_{r} \Rightarrow \max _{k \in N_{i}^{r}} x_{k} \leq \min _{N_{i}^{r}} \bar{x}_{k}\right] .
\end{aligned}
$$

Suppose that

$$
\begin{aligned}
& \max _{k \in N} \underline{x}_{k}>m_{1} \\
& \text { or } \\
& (\exists r \in S(A))\left(\exists i \in E^{r}\right)\left[\min _{k \in N_{i}^{r}} \bar{x}_{k}<m_{r} \wedge \max _{k \in N_{i}^{r}} \underline{x}_{k}>\min _{k \in N_{i}^{r}} \bar{x}_{k}\right] .
\end{aligned}
$$

Inequality (11) implies the existence of $l \in N$ such that $\underline{x}_{l}>m_{1}$. Then for each $x \in \mathbf{X}$ we get $x_{l}>m_{1}$. According to Lemma 3.1 there is no eigenvector of $A$ in $\mathbf{X}$. Thus $\mathbf{X}$ is not a possible eigenvector of $A$.

If condition (12) is fulfilled then there exists $r \in$ $S(A), i \in E^{r}$ such that $\min _{k \in N_{i}^{r}} x_{k} \leq \min _{k \in N_{i}^{r}} \bar{x}_{k}<m_{r}$ for each $x \in \mathbf{X}$. Moreover, the inequality $\max _{k \in N_{i}^{r}} \underline{x}_{k}>\min _{k \in N_{i}^{r}} \bar{x}_{k}$ implies the existence of $t, s \in N_{i}^{r}$ such that $x_{t} \geq \underline{x}_{t}=\max _{k \in N_{i}^{r}} \underline{x}_{k}>\min _{k \in N_{i}^{r}} \bar{x}_{k}=$ $\bar{x}_{s} \geq x_{s}$, i.e. $x_{t} \neq x_{s}$ for each $x \in \mathbf{X}$. In view of Theorem 3.2 there is no eigenvector $x \in \mathbf{X}$. Thus $\mathbf{X}$ is not a possible eigenvector of $A$.

For the converse implication suppose that

$\max _{k \in N} \underline{x}_{k} \leq m_{1}$ eigenvector of $A$.

and

$(\forall r \in S(A))\left(\forall i \in E^{r}\right)\left[\min _{k \in N_{i}^{r}} \bar{x}_{k}<m_{r} \Rightarrow \max _{k \in N_{i}^{r}} \underline{x}_{k} \leq \min _{N_{i}^{r}} \bar{x}_{k}\right]$.

We shall construct the vector $x^{*} \in \mathbf{X}$ such that $x^{*}$ is an eigenvector of $A$ by the following algorithm.

Algorithm $\mathscr{A}_{2}$ : Finding the eigenvector $x^{*}(A)$

Input: $A\left(a_{0}, a_{1}, \ldots, a_{n-1}\right)$, interval vector $\mathbf{X} \in B(n)$

Output: the sets $T^{j}$, the elements $x^{*}\left(T^{j}\right) \in B$, the numbers $r^{*}\left(T^{j}\right) \in S(A)$ and the vector $x^{*} \in B(n)$

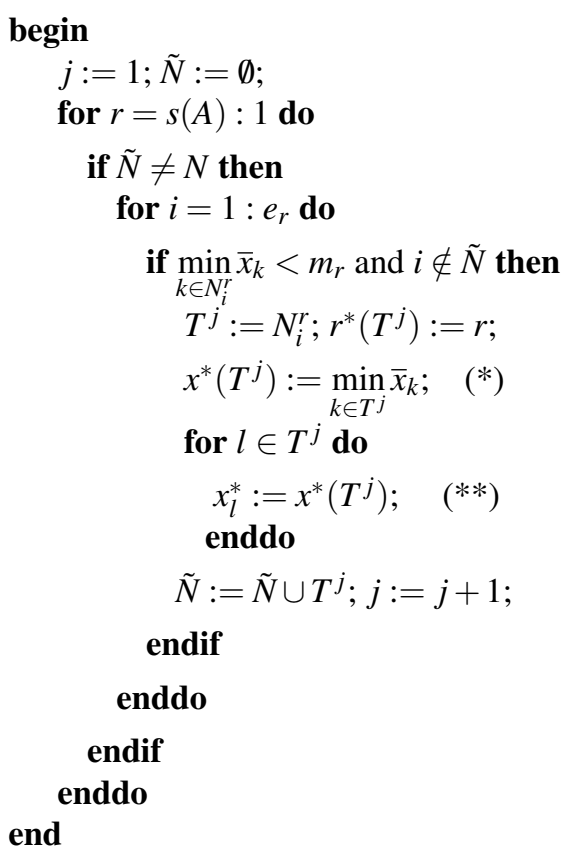

As the output of Algorithm $\mathscr{A}_{2}$ we get the sets $T^{1}, T^{2}, \ldots, T^{p}, p \leq n$. Similarly as in Algorithm $\mathscr{A}_{1}$, the sets $T^{1}, T^{2}, \ldots, T^{p}, p \leq n$ create the partition $\mathscr{T}$ on $N$. Moreover, the conditions $i$ ) $-i i i)$ of Theorem 3.1 are satisfied. The inequality $\max _{k \in N_{i}^{r}} \underline{x}_{k} \leq \min _{N_{i}^{r}} \bar{x}_{k}$ implies that $\min _{k \in N_{i}^{r}} \bar{x}_{k} \in$ $\left[\underline{x}_{l}, \bar{x}_{l}\right]$ for each $l \in N_{i}^{r}$. Thus the constructed vector $x^{*}$ lies in $\mathbf{X}$

In view of Theorem 3.1 the vector $x^{*}$ is an eigenvector of $A$. Moreover, $x^{*}$ is the greatest eigenvector in $\mathbf{X}$ because of $(*),(* *)$ and Theorem 3.2

\subsection{Universal eigenvectors}

Theorem 4.2. Let $A=A\left(a_{0}, a_{1}, \ldots, a_{n-1}\right)$ be a circulant matrix. An interval vector $\mathbf{X}$ is a universal eigenvector of $A$ if and only if $\underline{x}$ is an eigenvector of $A, \max _{k \in N} \bar{x}_{k} \leq m_{1}$ and for each $r \in S(A), i \in N$ such that $e_{r} \neq n$ and $\underline{x}_{i}<m_{r}$, the equality $\underline{x}_{i}=\bar{x}_{i}$ is fulfilled.

Proof. Suppose that

$$
\underline{x} \text { is not an eigenvector of } A \quad \text { or } \quad \max _{k \in N} \bar{x}_{k}>m_{1}
$$


or

$$
(\exists i \in N)(\exists r \in S(A))\left[\underline{x}_{i}<m_{r} \wedge e_{r} \neq n \wedge \underline{x}_{i} \neq \bar{x}_{i}\right] .
$$

If $\underline{x}$ is not an eigenvector of $A$ then $\mathbf{X}$ is not a universal eigenvector of $A$.

In the second case the inequality $\max _{k \in N} \bar{x}_{k}>m_{1}$ implies that $\bar{x}$ is not an eigenvector of $A$ and consequently $\mathbf{X}$ is not a universal eigenvector of $A$.

In the third case suppose that condition 15 is satisfied and $x$ is an eigenvector of $A$. Since $e_{r} \neq n$, there exists $j \in N, j \neq i$ such that $j \equiv i \bmod e_{r}$. Since $\underline{x}$ is an eigenvector of $A$, we have $\underline{x}_{i}=\underline{x}_{j}$. Define the vector $\tilde{x} \in B(n)$ as follows

$$
\tilde{x}_{k}= \begin{cases}\bar{x}_{k} & \text { for } k=i, \\ \underline{x}_{k} & \text { for } k \neq i .\end{cases}
$$

Since $\tilde{x}_{j}<m_{r}, i \equiv j \bmod e_{r}$ and $\tilde{x}_{i} \neq \tilde{x}_{j}$, the vector $\tilde{x}$ is not an eigenvector of $A$ by Lemma 3.2. Thus $\mathbf{X}$ is not a universal eigenvector of $A$.

For the converse implication suppose that $x$ is an eigenvector of $A, \max _{k \in N} \bar{x}_{k} \leq m_{1}$ and $\mathbf{X}$ is not a universal eigenvector of $A$. We will prove that the condition (15) is satisfied.

If $x \in \mathbf{X}$ is not an eigenvector of $A$ and $\max _{k \in N} \bar{x}_{k} \leq m_{1}$ then, by Theorem 3.2, there exist $r \in S(A)$ and $j \in E^{r}$ such that $\min _{k \in N_{j}^{r}} x_{k}<m_{r}$ and $x_{t} \neq x_{s}$ for some $t, s \in N_{j}^{r}$. Then $e_{r} \neq n$ and there exists $i \in N_{j}^{r}$ such that $\underline{x}_{i}=\min _{k \in N_{j}^{r}} \underline{x}_{k}<m_{r}$. The existence of $t, s \in N_{j}^{r}$ such that $x_{t} \neq x_{s}$ implies $x_{i} \neq x_{t}$ or $x_{i} \neq x_{s}$. Without lost of generality we shall suppose that $x_{i} \neq x_{t}$. Whereas $\underline{x}$ is an eigenvector of $A$ we get $\underline{x}_{i}=\underline{x}_{t}$. In the conjunction with the inequality $x_{i} \neq x_{t}$ we get $\underline{x}_{i} \neq \bar{x}_{i}$ or $\underline{x}_{t} \neq \bar{x}_{t}$. Thus the condition (15) is satisfied.

\section{Corollary 4.1.}

i) If $A=A\left(a_{0}, a_{1}, \ldots, a_{n-1}\right)$ is such that $P_{1}=\{0\}$ and $\left[\underline{x}_{i}, \bar{x}_{i}\right] \subseteq\left[m_{1}, m_{2}\right]$ for each $i \in N$ then $\mathbf{X}$ is a universal eigenvector of $A$.

ii) Let for each $i \in N$ the inequality $\underline{x}_{i}<m_{r^{*}}$, where $r^{*}=\min \left\{r ; e_{r} \neq n\right\}$, holds true. An interval vector $\mathbf{X}$ is a universal eigenvector of $A$ if and only if $\underline{x}=\bar{x}$ and $\underline{x}$ is an eigenvector of $A$.

iii) If there exists $j \in N$ such that $\underline{x}_{j}<m_{\tilde{r}}$ where $\tilde{r}=$ $\min \left\{r ; e_{r}=1\right\}$. Then $\mathbf{X}$ is a universal eigenvector of $A$ if and only if $\underline{x}_{i}=\bar{x}_{i}=\underline{x}_{j}$ for each $i \in N$.

\section{EXAMPLES}

Example 5.1. Check out, whether a given interval vector $\mathbf{X}$ is a possible (universal) eigenvector of $A$, if

$$
A=A(14,2,5,3,1,0,10,1,7,2,1,1)
$$

and

$$
\begin{gathered}
\mathbf{X}=([4,6],[6,8],[3,10],[5,7],[2,14],[5,12],[4,8],[2,9] \\
[3,9],[4,9],[4,10],[9,14])^{T}
\end{gathered}
$$

The strictly decreasing sequence of inputs is $M(A)=$ $\left(m_{1}, m_{2}, \ldots, m_{8}\right)=(14,10,7,5,3,2,1,0)$. The sets $P_{r}, r \in$ $\{1,2, \ldots, 8\}$ are $P_{1}=\{0\}, P_{2}=\{6\}, P_{3}=\{8\}, P_{4}=\{2\}$, $P_{5}=\{3\}, P_{6}=\{1,9\}, P_{7}=\{4,7,10,11\}, P_{8}=\{5\}$. We compute the numbers $e_{r}, r \in\{1,2, \ldots, 8\}$ using (3). We get $e_{1}=12, e_{2}=6, e_{3}=2, e_{4}=2, e_{5}=e_{6}=e_{7}=e_{8}=1$.

We have to check conditions (9) and (10). Denote by (I) the implication

$$
\min _{k \in N_{i}^{r}} \bar{x}_{k}<m_{r} \Rightarrow \max _{k \in N_{i}^{r}} x_{k} \leq \min _{N_{i}^{r}} \bar{x}_{k}
$$

from 10 .

Since $\max _{k \in N} x_{k}=9 \leq m_{1}$ inequality (9) holds true. To verify 10 we start with $r=s(A)=8$. We compute $e_{8}=1$, $E_{8}=\{1\}$ and $N_{1}^{8}=N$. Since $\min _{k \in N} \bar{x}_{k}=6 \geq 0$ the implication (I) is true for $r=8$.

In fact, it is sufficient to start with the least $r$ such that $e_{r}=1$, i.e., $r=5$. Since $\min _{k \in N} \bar{x}_{k}=6 \geq m_{5}$ the implication in (10) is true for $r \in\{5,6,7,8\}$.

Similarly, since $e_{3}=e_{4}=2$ we continue with $r=3$. We have $E_{3}=\{1,2\}$ and $N_{1}^{3}=\{1,3,5,7,9,11\}, N_{2}^{3}=$ $\{2,4,6,8,10,12\}$.

For $i=1$ we have $\min _{k \in N_{1}^{3}} \bar{x}_{k}=6<m_{3}$, so we compute $\max _{k \in N_{1}^{3}} x_{k}=4 \leq \min _{k \in N_{1}^{3}} \bar{x}_{k}$. Thus the implication (I) holds true. Moreover, we can construct the vector $x^{*}$ using Algorithm $\mathscr{A}_{2}$. We put $x_{1}^{*}=x_{3}^{*}=x_{5}^{*}=x_{7}^{*}=x_{9}^{*}=x_{11}^{*}=6$. We have $\tilde{N}=N_{1}^{3}$.

For $i=2$ we compute $\min _{k \in N_{2}^{3}} \bar{x}_{k}=8 \geq m_{3}$, hence the implication is true.

For $r=2$ we have $N_{1}^{2}=\{1,7\}, N_{2}^{2}=\{2,8\}, N_{3}^{2}=$ $\{3,9\}, N_{4}^{2}=\{4,10\}, N_{5}^{2}=\{5,11\}, N_{6}^{2}=\{6,12\}$. For $i \in\{1,3,5\}$ we have $i \in \tilde{N}$, so we consider only $i \in\{2,4,6\}$. We compute $\min _{k \in N_{2}^{2}} \bar{x}_{k}=8<m_{2}$ and $\max _{k \in N_{2}^{2}} x_{k}=6 \leq \min _{k \in N_{2}^{2}} \bar{x}_{k}$. We put $x_{2}^{*}=x_{8}^{*}=8$.

For the set $N_{4}^{2}$ we get $\min _{k \in N^{2}} \bar{x}_{k}=7<m_{2}$ and $\max _{k \in N^{2}} x_{k}=$ $5 \leq \min _{k \in N_{4}^{2}} \bar{x}_{k}$. We put $x_{4}^{*}=x_{10}^{*}=7$.

Since $\min _{k \in N_{6}^{2}} \bar{x}_{k}=12 \geq m_{2}$, the implication (I) is true.

For $r=1$ we have $N_{i}^{1}=\{i\}$ for each $i \in N$. In this case the implication always holds true. It remains to put $x_{6}^{*}=12, x_{12}^{*}=14$.

The interval vector $\mathbf{X}$ is not a universal eigenvector of $A$, because the vector $\underline{x}$ is not an eigenvector of $A$.

\section{Answer:}

- The interval vector $\mathbf{X}$ is a possible eigenvector of $A$ and the vector $x^{*}=(6,8,6,7,6,12,6,8,6,7,6,14)^{T}$ is the greatest eigenvector of $A$ lying in $\mathbf{X}$.

- The interval vector $\mathbf{X}$ is not a universal eigenvector of $A$.

Example 5.2. In this example we show three universal eigenvectors of the matrix A(14,2,5,3,1,0,10,1,7,2,1,1) from the previous example using Corollary 4.1 . 
i) The given matrix satisfies the condition $P^{1}=\{0\}$. We take the vector $\mathbf{X}$ such that $\left[\underline{x}_{i}, \bar{x}_{i}\right] \subseteq\left[m_{1}, m_{2}\right]=$ $[10,14]$ for each $i \in N$. This condition is satisfied, e.g., by interval vector

$\mathbf{X}=([12,14],[11,13],[10,14],[11,12],[13,13],[10,12]$,

$$
[12,13],[11,12],[10,13],[11,11],[12,14],[11,12]) .
$$

In view of Corollary 4.1 ) the vector $\mathbf{X}$ is a universal eigenvector of $A$.

ii) To demonstrate the use of Corollary 4.1 i), let us set $\underline{x}=(5,7,5,7,5,8,5,7,5,7,5,8)^{T}$. The vector $\underline{x}$ is an eigenvector of $A$ according to Theorem 3.2 . Since $r^{*}=2$ and $\underline{x}_{i}<m_{2}=10$ for each $i \in N$, the only universal eigenvector $\mathbf{X}$ is the constant vector, i.e., $\bar{x}=\underline{x}$.

iii) We have $\tilde{r}=5, m_{\tilde{r}}=3$. Let $\underline{x}_{1}=2$. According to Corollary 4.1 iii), the only universal eigenvector of $A$ is such that $\underline{x}_{i}=\bar{x}_{i}=2$ for each $i \in N$.

\section{REFERENCES}

[1] CECHLÁROVÁ, K.: Eigenvectors in bottleneck algebra, Lin. Algebra Appl. 175 (1992), 63-73.

[2] FIEDLER, M. - NEDOMA, J. - RAMÍK, J. - ROHN, J. - ZIMMERMANN, K.: Linear Optimization Problems with Inexact Data, Springer-Verlag, Berlin 2006.

[3] GAVALEC, M.: Monotone eigenspace structure in max-min algebra, Linear Alg. Appl. 345 (2002), 149167.

[4] GAVALEC, M. - PLAVKA, J.: Monotone interval eigenproblem in max-min algebra, Kybernetika 46 (3) (2010), 387-396.

[5] GAVALEC, M. - TOMÁŠKOVÁ, H.: Eigenspace of a circulant max-min matrix, Kybernetika 46(3) (2010), 397-404.

[6] GODRAN, M. - MINOUX, M.: Graphs, dioids and semirings: new models and algorithms, Springer 2008.
[7] HORVATH, T. - VOJTÁŠ, P.: Induction of fuzzy and annotated logic programs, Inductive Logic Programming 4455 (2007) 260-274.

[8] MYŠKOVÁ, H.: Interval systems of max-separable linear equations, Linear Alg. Appl. 403 (2005), 263272.

[9] MYŠKOVÁ, H.: Control solvability of interval systems of max-separable linear equations, Linear Alg. Appl. 416 (2006), 215-223.

[10] PLAVKA, J.: Eigenproblem for circulant matrices in max-algebra, Optimization 50 (2001), 477-483.

[11] PLAVKA, J.: I-parametric eigenproblem in max algebra, Discrete Applied Mathematics 150 (2005), 1628.

[12] PLAVKA, J. - SZABÓ, P.: On the $\lambda$-robustness of matrices over fuzzy algebra, Discrete Applied Mathematics 159 (5) (2011), 381-388.

[13] SANCHEZ, E.: Resolution of eigen fuzzy sets equations, Fuzzy Sets and Systems 1 (1978), 69-74.

[14] SANCHEZ, E.: Medical diagnosis Medical diagnosis and composite relations, In: M. M. Gupta, R. K. Ragade, R. R. Yager, Eds, Advances in Fuzzy Set Theory and Applications (North- Holland, Amsterdam- New York, 1979), 437-444.

[15] ZIMMERMANN, K.: Extremální algebra, Ekonomicko-matematická laboratoř Ekonomického ústavu ČSAV, Praha, 1976.

Received July 27, 2012, accepted September 28, 2012

\section{BIOGRAPHY}

Helena Myšková was born on 14.1. 1964. She graduated (RNDr) with distinction at the Faculty of Science at P. J. Šafárik University in Košice in 1987. She defended her $\mathrm{PhD}$ in the field of discrete mathematics in 2009; her thesis title was "Solving of systems and interval systems of linear equations over the max-plus and max-min algebra". She is working as a tutor at the Department of Mathematics and Theoretical Informatics since 1995. Her scientific research is focusing on interval computations in extremal algebras. 